\title{
1 Conserved and non-conserved triggers of 24-nt reproductive phasiRNAs in
}

\section{2 eudicots}

3

4 Suresh Pokhrel ${ }^{1,2}$, Kun Huang ${ }^{3}$, and Blake C. Meyers ${ }^{1,2^{*}}$

5

$6 \quad{ }^{1}$ Donald Danforth Plant Science Center, Saint Louis, MO 63132

$7 \quad{ }^{2}$ Division of Plant Sciences, University of Missouri-Columbia, Columbia, MO 65211

$8{ }^{3}$ Delaware Biotechnology Institute and Department of Plant and Soil Sciences, University of

9 Delaware, Newark, Delaware 19711

*Corresponding author: bmevers@danforthcenter.org

Abstract

14 Plant small RNAs (sRNAs) play important roles in plant growth and development by modulating expression of genes and transposons. In many flowering plant species, male reproductive

16 organs, the anthers, produce abundant phased small interfering RNAs (phasiRNAs). Two classes

17 of reproductive phasiRNAs are generally known, mostly from monocots: pre-meiotic 21-nt

18 phasiRNAs triggered by miR2118, and meiotic 24-nt phasiRNAs triggered by miR2275. Here, we

19 describe conserved and non-conserved triggers of 24-nt phasiRNAs in several eudicots. We

20 found that the abundant 24-nt phasiRNAs in the basal eudicot columbine are produced by the

21 canonical trigger, miR2275, as well as by other non-conserved triggers, miR482/2118 and

22 aco_cand81. These triggering miRNAs are localized in microspore mother cells (MMC) and

23 tapetal cells of meiotic and post-meiotic stage anthers. Furthermore, we identified a new

24 trigger (miR11308) of 24-nt phasiRNAs and an expanded number of 24-PHAS loci in wild

25 strawberry. We validated the presence of miR2275-derived 24-nt phasiRNAs pathway in rose.

26 Finally, we evaluated all the eudicots that have been validated for the presence of 24-nt

27 phasiRNAs as models to study biogenesis and function of 24-nt phasiRNAs and conclude that

28 columbine would be an excellent model because of its extensive number of 24-PHAS loci and its 29 diversity of trigger miRNAs. 


\section{Introduction}

32 Plant sRNAs range from 20 to 24 nucleotides (nt) in length and are mainly classified into three

33 groups based on their origin and biogenesis. The groups are (1) microRNAs (miRNAs), (2)

34 heterochromatic small interfering RNAs (hc-siRNAs), and (3) phasiRNAs (Fei et al., 2013). Plant

35 SRNA biogenesis requires RNA-DEPENDENT RNA POLYMERASE (RDR), DICER-LIKE (DCL), and

36 Argonaute (AGO), proteins, plus other accessory proteins. All types of sRNAs associate with

37 AGO proteins to initiate both transcriptional and post-transcriptional silencing via formation of

38 RNA-induced silencing complexes. Among these sRNA classes, phasiRNAs are generated by the

39 action of 22-nt miRNA triggers on messenger RNA (mRNA) targets derived from RNA

40 Polymerase II; the target is subsequently processed by RDR6 and DCL proteins into 21- or 24-nt

41 siRNAs (Fei et al., 2013).

43 Reproductive 24-nt phasiRNAs were reported mainly in the male organs of grasses (Poaceae)

44 (Zhai et al., 2015b; Song et al., 2012; Johnson et al., 2009; Patel et al., 2020), and more recently

45 in six non-grass monocots (Patel et al., 2020) and eight eudicots (Xia et al., 2019). This size class

46 is abundant in the meiotic stage of anthers and are mainly triggered by miR2275, although

47 there are cases of trigger-less 24-nt phasiRNA-producing loci (aka "24-PHAS" loci) in tomato and

48 petunia (Xia et al., 2019), and in monocots outside the Poaceae (Kakrana et al., 2018; Patel et

49 al., 2020). The spatiotemporal pattern of 24-nt phasiRNAs in male reproductive tissues has

50 been well-described in maize, rice, and litchi (Zhai et al., 2015b; Song et al., 2012; Xia et al.,

51 2019). The loci that generate the 24-nt phasiRNAs, known as 24-PHAS loci, correspond to

52 unique or low-copy genomic regions which makes 24-nt phasiRNAs different from 24-nt

53 heterochromatic siRNAs (hc-siRNAs) that function to suppress transposons (Zhai et al., 2015a).

54 These reproductive 24-nt phasiRNAs lack obvious targets but play a role in male fertility, as

55 their absence due to a knockout of DCL5 in maize produces temperature-sensitive genic male

56 sterility (Teng et al., 2020). The 24-nt reproductive phasiRNAs may be comparable or analogous

57 to animal piRNAs (PIWI-interacting RNAs) which also play a role in male fertility (Kotov et al.,

58 2019). The role of animal piRNAs is not entirely clear and is an active topic of investigation by 
59 numerous labs; however, at least some piRNAs function as a defense mechanism to protect genetic information in germ cells from transposons (Kotov et al., 2019).

62 Recently, we identified 24-nt phasiRNAs in seven eudicots, demonstrating the broad presence 63 of the miR2275/24-nt phasiRNA pathway in angiosperms (Xia et al., 2019). The general lack of

64 information on this pathway in eudicots motivated us study more species and in more detail.

65 Here, we report the existence of the 24-nt phasiRNA pathway in the basal eudicot columbine (Aquilegia coerulea), expand the number of known 24-PHAS loci in wild strawberry (Fragaria vesca), and validate the pathway in rose (Rosa chinensis). We identify triggers of 24-nt phasiRNAs other than miR2275, in columbine and wild strawberry. Finally, we evaluated the eudicot species validated for the presence of the 24-phasiRNA pathway by potential characteristics, with the aim to identify a model species in which to study biogenesis and

71 function of this pathway; we conclude that columbine would be an excellent model for these 72 studies.

\section{Results}

Conserved triggers initiate 24-nt phasiRNAs in columbine anthers

76 miR2275 is mostly conserved in angiosperms and is the only trigger of 24-nt phasiRNAs known

77 to date (Xia et al., 2019). Using this as a marker of the 24-nt phasiRNA pathway, we searched

78 for the presence of miR2275 in the genome of columbine and identified 11 clusters of miR2275

79 precursors that yield 19 mature duplexes with 12 unique, expressed, miR2275 sequences

80 (Figure 1A, Supplemental Table 1). Out of the 11 clusters, six are polycistronic precursors;

81 Figure 1B shows an example of a polycistronic cluster. To study 24-nt phasiRNA expression in

82 columbine, we analyzed sRNAs data derived from vegetative tissues (leaf and root) and

83 reproductive tissues (four unopened bud stages; Pokhrel et al., 2020). We found that mature

84 miR2275 sequences highly accumulate in bud stages two and three (Figure 1C) which represent

85 meiotic/post-meiotic stages of anther development. We identified an extensive number (642)

86 of 24-PHAS loci with miR2275 target sites as the only apparently conserved sequence among

87 the precursors (Supplemental Figure 1A, Supplemental Table 2). The 24-nt phasiRnAs spawned 
88 from these precursors demonstrate similar patterns of abundance as their triggers, suggesting a

89 tight temporal regulation as in grasses and litchi (Figure 1D; Zhai et al., 2015b; Xia et al., 2019).

90 The miR2275-triggered 24-PHAS loci are highly phased and the first 24-nt phasiRNA originates

91 from cleavage site indicated by the red arrow in an example locus as shown (Supplemental

92 Figure 1B).

Non-conserved triggers initiate 24-nt phasiRNAs in columbine anthers

95 During our analysis of possible triggers of 24-PHAS loci, we unexpectedly found that both (i) a previously-unannotated, apparently lineage-specific miRNA (aco_cand81; Supplemental Figure 2A) and (ii) miR2118 family members (Supplemental Figure 2B) triggered production of 24-nt phasiRNAs from 15 and 22 loci, respectively (Figure 2A, 2B). The expression profile of the 24-nt phasiRNAs generated via the non-conserved triggers are similar to those derived from the conserved, canonical trigger, miR2275 (Figure 1D, 2A, 2B). Ten out of 15 24-PHAS loci targeted

101 by miRNA aco_cand81 are derived from a cluster spanning $32.2 \mathrm{~kb}$ on chromosome 4

102 (Supplemental Figure 2C, Supplemental Table 2), suggesting that these 24-nt phasiRNAs were

103 selectively retained in the genome as the loci don't resemble the product of a recent tandem

104 duplication -- a whole precursor alignment of these loci shows no conservation at the

105 nucleotide level, except for the target site. That is, the only conserved sequence between these

10615 24-PHAS loci is the target site of aco_cand81 (Figure 2C). Similarly, the 22 24-PHAS loci

107 triggered by miR2118 also showed conservation of only the target site (Figure 2D, Supplemental

108 Table 2). In the genome browser, it is apparent that the first 24-nt phasiRNAs are generated

109 from the cleavage site of aco_can81 and miR2118 (Figure 2E and 2F), respectively. Interestingly,

110 members of both of these miRNA families also trigger production of 21-nt reproductive

111 phasiRNAs in columbine (Pokhrel et al., 2020).

113 In the genome of columbine, 24-PHAS loci are distributed across the arms of all chromosomes,

114 while one arm of chromosome 4 has a particularly high concentration of loci (313 loci out of

115679 total loci; outer circle, Figure 3A). We examined the strand-specificity of 24-PHAS

116 precursors (inferring the Pol II-derived strand from the target site match) within the 
117 chromosome 4 clusters. We found that a conservation of strandedness within a cluster,

118 meaning that all the precursors are encoded on the same chromosomal strand; however, there

119 is again no conservation of nucleotide sequence other than the target site of the triggering

120 miRNAs, even within these tandemly arranged sequences. We hypothesized that there was a

121 duplication of 24-PHAS loci that caused the arrangement (Supplemental Figure 1A; Figure 3B).

122 The duplications must have occurred long enough ago to allow nearly complete sequence

123 divergence; a dot plot of 24-PHAS loci from chromosome 4 demonstrates very few similarities

124 among these loci (Supplemental Figure 2D). Chromosome 4 of Aquilegia has previously been

125 shown to have a "distinct evolutionary path from the rest of the genome" (Aköz and Nordborg,

126 2019), and containing many duplications consistent with the arrangement of the 24-PHAS loci.

127 In contrast, the conserved and non-conserved triggers of these phasiRNAs are broadly

128 distributed, on all chromosomes except 2 and 7 (inner circle, Figure 3A).

We next profiled the single nucleotide compositions of 24-nt phasiRNAs from columbine,

131 analyzed in three different groups based on their triggers, and compared these to hc-siRNAs.

132 This approach can identify seemingly minor but statistically significant differences in SRNA

133 populations (Patel et al., 2018a). We computed the frequency of each nucleotide (A, C, G and U)

134 at each position (Supplemental Figure 3) for the 24-nt phasiRNAs and hc-siRNAs. We observed

135 position-specific biases, indicating different nucleotide composition, in these two different

136 classes of siRNAs (Supplemental Figure 3A and B). Moreover, we found that the nucleotide

137 composition of 24-nt phasiRNAs derived from different triggers was the same (Supplemental

138 Figure $3 \mathrm{C}$ ), consistent with shared origins and derivations.

140 Spatiotemporal localization of triggers and 24-nt phasiRNAs in columbine

141 We next examined the spatiotemporal localization of all three reproductive phasiRNA triggers

142 in pre-meiotic, meiotic, and post-meiotic stages of columbine anther development. The non-

143 conserved triggers aco_can81 and miR2118 were enriched compared to miR2275, in all stages.

144 The triggers localized to all cell layers and were enriched in the microspore mother cells (MMC)

145 of pre-meiotic stage or tapetal cells of meiotic/post-meiotic stages, suggesting 24-nt phasiRNAs 
originate in those cell layers (Figure 4A). Because the imaging method we utilized is a single-

147 molecule, quantitative method, we also quantified the copy number of each phasiRNA trigger in

$148 \mathrm{MMC}$, tapetal cells, and other cell layers. Overall, miR2275 was enriched in the post-meiotic 149 stage compared to other stages, possibly reflecting additional roles it may play in this stage, 150 while miR2118 and aco_cand81 were enriched in meiotic/post-meiotic stages, mirroring the 151 abundance patterns of the 24-nt phasiRNAs they generate (Figure 4B; Figure 2A and 2B). The

152 spatiotemporal pattern of triggering molecules in columbine is consistent with that of litchi and 153 maize (Xia et al., 2019; Zhai et al., 2015b), consistent with a conserved and possibly important 154 regulatory role of these RNAs in male gamete formation.

Conserved and non-conserved triggers of 24-nt phasiRNAs in the Rosaceae and Magnoliids

157 Recently, we described the presence of a miR2275-triggered, 24-nt phasiRNA pathway in wild 158 strawberry (Xia et al., 2019) with the highest known number (which was 221) of 24-PHAS loci in 159 eudicots. By using two types of phasiRNA-identifying software, coupled with extensive 160 experimental data (Xia et al., 2019), we aimed to saturate the identification of 24-PHAS loci in 161 wild strawberry. The result was that this analysis expanded the set of known miR2275-triggered 162 24-PHAS loci to 326 in total (Supplemental Figure 4A, Supplemental Table 3). These phasiRNAs 163 were expressed mostly in anther stages 7 to 9, corresponding to the meiotic stages of anther 164 development. The expression pattern of miR2275 variants also followed a similar pattern 165 (Supplemental Figure 4B, 4C). Then, we sought to determine if any additional triggers were 166 previously missed, as prior work focused only on miR2275; we found that miR11308 generated 167 24-nt phasiRNAs from six 24-PHAS loci, while miR482/2118 family members targeted four 24168 PHAS loci (Figure 5A, Supplemental Table 3). The expression pattern of 24-nt phasiRNAs 169 generated via these non-conserved triggers was similar to that of the canonical trigger, 170 miR2275 (Figure 5A; Supplemental Figure 4A). The target site of miR11308 was the only 171 conserved region in six 24-PHAS loci (Figure 5B). These loci mostly originated from arms of all 172 chromosomes and their triggers are encoded by loci found on chromosomes 1, 4, 5 and 6 173 (Figure 5C). 
175 To investigate the conservation of this pathway, we analyzed SRNA data of vegetative tissues,

176 and anthers from four unopened bud stages of rose (Guo et al., 2019; Pokhrel et al., 2020). We

177 found 112 24-PHAS loci triggered by miR2275 variants, accumulating to the greatest abundance

178 at bud stage one (Figure 5D, Supplemental Table 4). Genomic searches for the presence of

179 miR2275 in rose identified three clusters of precursors, two of which were apparently

180 polycistronic; an example locus is shown in Supplemental Figure 5A (see also Supplemental

181 Table 5). We observed the expression of seven mature sequences of miR2275, and their

182 abundance patterns were similar to that of 24-nt phasiRNAs (Supplemental Figure 5B and 5C).

183 We found no evidence for the non-conserved triggers of strawberry (i.e. miR2118/42 and

184 miR11308) targeting 24-PHAS loci in rose, perhaps due to species-specific variation, or possibly

185 due to inadequate sampling of anther stages. Similar to wild strawberry, the PHAS loci are

186 distributed across arms of all chromosomes, while the miRNA triggers originated from

187 chromosomes 2 and 5 (Figure 5E).

189 We previously characterized the presence of miR2275 loci in 209 genomes of flowering plants

190 spanning different clades of eudicots and monocots (Xia et al., 2019); however, that analysis

191 excluded the magnoliids as there were no sequenced genomes at that time. Therefore, we

192 investigated a recently published sequence genome of a magnoliid species, Magnolia biondii,

193 (Dong et al., 2020). We found nine unique mature miR2275 sequences derived from 11

194 precursors (Supplemental Figure 6A), three of which appear to be polycistronic precursors, an

195 example locus is shown in Supplemental Figure 6B (see also Supplemental Table 5).

197 Evaluation of eudicot species as models to study 24-nt phasiRNAs

198 In total, from this and prior work, the production of 24-nt phasiRNAs was validated in

199 reproductive tissues of ten eudicots (Table 1). To enable further insights into potential

200 mechanistic roles of these phasiRNAs in reproduction, we sought to identify potential model

201 species for future work. Thus, we compared different characteristics such as genome size, plant

202 life cycle, the number of 24-PHAS loci and miRNA triggers, and the number of genomic DCL3

203 copies of the validated eudicot species. Of these species, transformation by tissue culture has 
204 been widely reported for all but columbine. Based on this comparison, we conclude that

205 columbine would be an excellent model system due to its phylogenetic position among

206 flowering plants as a basal eudicot, its diversity of trigger miRNAs, and its extensive number of

207 24-PHAS loci. Although it lacks an efficient transformation system, the recent use of

208 developmental genes encoding GROWTH-REGULATING FACTORS such as AtGRF5 or TaGRF4

209 may help to generate genetically modified plants in recalcitrant eudicots such as columbine

210 (Kong et al., 2020; Debernardi et al., 2020). From the perspective of having an already

211 established transformation system and its ease of propagation, wild strawberry may be a good

212 model system, as it also has a diversity of triggers and extensive 24-PHAS loci, though fewer

213 compared to columbine.

\section{Discussion}

216 We showed that the 24-nt reproductive phasiRNA pathway is conserved in a basal eudicot,

217 columbine. Recently, we hypothesized that this pathway emerged coincident with angiosperms

218 with selective maintenance or loss in some flowering plant lineages (Xia et al., 2019). This was

219 based in part on the presence/absence of a canonical pathway trigger (miR2275). The basal

220 angiosperms Amborella trichopoda and Nymphaea colorata are the only species outside of

221 eudicots and monocots that have the trigger encoded in their genomes but lack bona fide 24-

222 PHAS loci (Xia et al., 2019; Patel et al., 2020). Our confirmation of the presence of this pathway

223 in columbine, an early diverging lineage of the eudicots, provides strong support for the

224 emergence of the pathway prior to the emergence of the core eudicots. Furthermore, in

225 addition to wild strawberry, we validated the presence of this pathway in another Rosaceae

226 family member, rose, providing additional evidence for widespread conservation of this

227 pathway. We also identified the presence of miR2275 in Magnolia biondii, a magnoliid,

228 suggesting an origin for this pathway of at least 150 MYA in core angiosperms (Figure 6A).

230 Surprisingly, we found lineage-specific triggers that generate 24-nt phasiRNAs in both

231 columbine and wild strawberry, distinct from the known, canonical trigger, miR2275. miR2275

232 is deeply conserved in angiosperms, including the characteristic of its polycistronic precursors, 
233 and the only known role of miR2275 is the generation of reproductive 24-nt phasiRNAs (Xia et

234 al., 2019). Interestingly, a well-studied miRNA, miR2118/482, known for triggering 21-nt

235 phasiRNAs from both protein-coding and non-coding loci in eudicots and monocots (Zhai et al.,

236 2015b; Bélanger et al., 2020; Pokhrel et al., 2020), has a function in generating 24-nt phasiRNAs

237 in columbine and wild strawberry, adding to the data from Solanaceous species (Xia et al.,

238 2019), barley and wheat (Bélanger et al., 2020) that there are multiple mechanisms for

239 activation of the 24-PHAS pathway independent of miR2275. However, there were few 24-PHAS

240 loci triggered by miR2118/482 compared to the total number 24-PHAS loci in each species.

241 miRNAs that may be lineage-specific, aco_cand81 in columbine and miR11308 in wild

242 strawberry, trigger both 24- and 21-nt reproductive phasiRNAs (Pokhrel et al., 2020). The 21-nt

243 phasiRNAs are produced by the action of DCL4 while DCL3 and DCL5 are responsible for 24-nt

244 phasiRNA production in eudicots and monocots, respectively. DCL5 resulted from a monocot-

245 specific duplication of DCL3 and is specialized for the production of reproductive 24-nt

246 phasiRNAs in monocots (Teng et al., 2020; Patel et al., 2020). Although DCL3 is single copy in

247 the genome of many eudicots, some eudicot lineages that have a duplicated copy of $D C L 3$ are

248 also predicted or validated for the presence of 24-nt phasiRNAs, perhaps consistent with a role

249 for the duplicated DCL3 copy in the biogenesis of reproductive 24-nt phasiRNAs (Pokhrel et al.,

250 2020; Xia et al., 2019). An interesting question for future work will be how different Dicer

251 proteins are recruited by the action of these different trigger miRNAs. Another interesting topic

252 for future work is the role of the polycistronic nature of the precursors observed for miRNA

253 triggers of both 21 and 24-nt reproductive phasiRNAs -- the conserved nature of this

254 characteristic suggests a functional role in reproductive phasiRNA biogenesis. Yet another topic

255 for investigation builds on our observations about the unusual concentration of reproductive

256 PHAS loci on chromosome 4 of columbine. Our data show that the 24-PHAS loci have

257 undergone extensive tandem and segmental duplications on chromosome 4. Since the 24-PHAS

258 loci are not repetitive DNA, and there are relatively few other genes interspersed among the

259 large number of 24-PHAS loci, it is entirely possible that this unusual chromosomal structure

260 and distinct evolutionary path of chromosome 4 (Aköz and Nordborg, 2019) has been driven by

261 and is the consequence of expansion in the 24-PHAS loci. 
263 We conclude with a model describing how different triggers produce 24-nt phasiRNAs in

264 different species of angiosperms (Figure 6B); this includes the hypothesis that the activity of the

265 lineage-specific miRNA triggers neofunctionalized to allow targeting precursors of both 21- and

266 24-nt reproductive phasiRNAs.

268 Taken together, these data indicate that conservation of 24-nt reproductive phasiRNAs in 269 eudicots is widespread, that they originated coincident with the evolutionary emergence of 270 angiosperms, and that diversification in their mode of biogenesis has occurred. Although the

271 functions of these 24-mers are still unclear, as they lack obvious target genes (Patel et al., 272 2018b), mutations in a pathway gene, $D C L 5$, in maize produced temperature sensitive male

273 sterility, consistent with important regulatory roles for these sRNAs in reproductive

274 development. Future studies are needed to advance our mechanistic understanding of these

275 sRNAs. Based on its phylogenic position as an early-diverged eudicot, the diversity of the miRNA

276 triggers, and the extensive number of 24-PHAS loci, we propose that columbine could serve as

277 an ideal model species with which to study these phasiRNAs in eudicots; it lacks only a facile

278 transformation system.

281 Small RNA datasets

282 The sRNA-seq data used in this study were published previously (Pokhrel et al., 2020; Xia et al., 283 2019; Guo et al., 2019; Xia et al., 2015). Briefly, in those studies, sRNA-seq was carried out on 284 vegetative tissues, unopened flower buds/anthers of columbine, rose, and wild strawberry.

\section{Data analysis}

287 SRNA-seq data analysis was performed as described (Pokhrel et al., 2020). Briefly, an in-house 288 preprocessing pipeline (Mathioni et al., 2017) was used to process the raw reads. Saturating 289 annotation of PHAS loci in all of the species was attempted by the combined use of two 290 software PHASIS (Kakrana et al., 2017) and ShortStack (Johnson et al., 2016). The PHAS loci 
291 from these two different software packages were merged using BEDTools (Quinlan and Hall,

292 2010). Target prediction was carried out using SPARTA (Kakrana et al., 2014) and the small RNA

293 abundances and phasing scores were viewed on the Meyers lab genome browser (Nakano et

294 al., 2020) to filter out the false-positive PHAS loci. Only PHAS loci having conserved target sites

295 for miRNA triggers were annotated as valid. miR2275 variants from columbine were aligned

296 with genome of Magnolia biondii using bowtie (Langmead et al., 2009) with default parameters

297 except $-v 2$. The resulting mapped positions were padded by $100 \mathrm{bp}$ on each side, and the

298 sequences were extracted using BEDTools (Quinlan and Hall, 2010); RNAfold (Hofacker, 2003)

299 was used to assess the folding of the sequences to identify the most likely precursors of

300 miR2275 encoded in the genome (Supplemental Table 5).

302 Data visualization

303 Multiple sequence alignment of strand-specific sequences from PHAS loci was performed using 304 MUSCLE (Edgar, 2004) with default parameters. The resulting alignment was viewed using 305 Jalview (Waterhouse et al., 2009). Sequences extracted from the alignment were used to 306 construct a dot plot of 24-PHAS loci from chromosome 4 in columbine using D-Genies

307 (Cabanettes and Klopp, 2018). Position-specific nucleotide biases were calculated using 308 PWM_StatisicalModel (Patel et al., 2018b). Circular plots were made using OmicCircos (Hu et 309 al., 2014) for the chromosomal distributions. In R, pheatmap (https://rdrr.io/cran/pheatmap/) 310 and ggpubr (https://github.com/kassambara/ggpubr) were used to draw heatmaps and box311 plot. The phylogenetic tree was derived from the TimeTree database (Kumar et al., 2017) and 312 modified using FigTree (http://tree.bio.ed.ac.uk/software/figtree/).

314 sRNA imaging

315 miRNA triggers aco_cand81d, miR2275e, and miR2118b were detected in anther sections using 316 sRNA-Exchange-PAINT as described in the published protocol (Huang et al., 2020). Probes for 317 miRNAs were designed using the VARNISH website (https://wasabi.ddpsc.org/ apps/varnish/).

318 Probes and corresponding imager strands with AlexFluor647 were ordered from IDT (Integrated 319 DNA Technologies, Inc.; sequences in Supplemental Table 6). Paraffin-embedded samples of 
320 carefully-staged flower buds were sectioned and dried on Wide Spectral Band 600+/- $100 \mathrm{~nm}$

321 Gold Fiducials coverglasses (part \#600-200AuF; Hestzig LLC, Leesburg, VA). A self-assembled

322 perfusion system consisted of a DH40iL culture dish incubate system (model 640388; Warner

323 Instruments LLC, Hamden, CT) and a quick release magnetic chamber for $25 \mathrm{~mm}$ low profile,

324 round coverslips (model 641943; Warner Instruments LLC). Before each imaging session, $1 \mathrm{ml}$

325 imager strand was pipetted in. Between each image session, samples were washed three times

326 with buffer C (1× PBS, $500 \mathrm{mM} \mathrm{NaCl}$, pH 8). Images were taken using a Dragonfly Spinning Disk

327 and Super Resolution Microscope (Andor, Oxford Instruments) using a 63x oil objective and a

328 Zyla camera at $100 \mathrm{~ms}$ exposure time. Image reconstruction and quantification were carried out

329 using Picasso software following the published protocol (Schnitzbauer et al., 2017). In brief,

3302000 frames of the each TIFF movie file were imported and processed under "Picasso: Localize"

331 using Min. Net Gradient of 1000. For quantification analysis using "Picasso: Render", we picked

33230 pixel diameter circle for each cell layer, which corresponding to the size of the cell in tested

333 aquilegia anther samples. For each sample, 10 locations were taken for each cell layer were

334 used to calculate the number of binding sites.

Acknowledgements

337 We thank members of the Meyers lab for helpful discussions, and Joanna Friesner for

338 assistance with editing. We thank Mayumi Nakano for assistance with data handling and Parth

339 Patel for help in the analysis of position-specific sequence variation. This work was supported

340 by resources from the Donald Danforth Plant Science Center and the University of Missouri-

341 Columbia. This research was supported by US NSF IOS award 1754097 to B.C.M., with additional

342 support from funds from the Donald Danforth Plant Science Center and the University of

343 Missouri - Columbia, plus the William H. Danforth Plant Science Fellow Award (to S.P.). 
References

Aköz, G. and Nordborg, M. (2019). The Aquilegia genome reveals a hybrid origin of core eudicots. Genome Biol. 20:256.

Bélanger, S., Pokhrel, S., Czymmek, K., and Meyers, B.C. (2020). Premeiotic, 24-nucleotide reproductive phasiRNAs are abundant in anthers of wheat and barley but not rice and maize. Plant Physiol. 184: 1407-1423.

Cabanettes, F. and Klopp, C. (2018). D-GENIES: Dot plot large genomes in an interactive, efficient and simple way. PeerJ 2018: e4958.

Debernardi, J.M., Tricoli, D.M., Ercoli, M.F., Hayta, S., Ronald, P., Palatnik, J.F., and transgenic plants. Nat. Biotechnol. 38: 1274-1279.

Dong, S. et al. (2020). The genome assembly and annotation of Magnolia biondii Pamp., a phylogenetically, economically, and medicinally important ornamental tree species. bioRxiv: 2020.06.17.158428.

Edgar, R.C. (2004). MUSCLE: A multiple sequence alignment method with reduced time and space complexity. BMC Bioinformatics 5: 113.

Fei, Q., Xia, R., and Meyers, B.C. (2013). Phased, Secondary, Small Interfering RNAs in Posttranscriptional Regulatory Networks. Plant Cell 25: 2400-2415.

Guo, J., Wang, Q., Liu, L., Ren, S., Li, S., Liao, P., Zhao, Z., Lu, C., Jiang, B., Sunkar, R., and

Hofacker, I.L. (2003). Vienna RNA secondary structure server. Nucleic Acids Res. 31: 3429-3431.

Hu, Y., Yan, C., Hsu, C.H., Chen, Q.R., Niu, K., Komatsoulis, G.A., and Meerzaman, D. (2014). Omiccircos: A simple-to-use R package for the circular visualization of multidimensional

372 Johnson, C., Kasprzewska, A., Tennessen, K., Fernandes, J., Nan, G.L., Walbot, V., Sundaresan, 
V., Vance, V., and Bowman, L.H. (2009). Clusters and superclusters of phased small RNAs in the developing inflorescence of rice. Genome Res. 19: 1429-1440.

Johnson, N.R., Yeoh, J.M., Coruh, C., and Axtell, M.J. (2016). Improved placement of multimapping small RNAs. G3 Genes, Genomes, Genet. 6: 2103-2111.

Kakrana, A. et al. (2018). Plant 24-nt reproductive phasiRNAs from intramolecular duplex mRNAs in diverse monocots. Genome Res. 28: 1333-1344.

Kakrana, A., Hammond, R., Patel, P., Nakano, M., and Meyers, B.C. (2014). SPARTA: A parallelized pipeline for integrated analysis of plant miRNA and cleaved mRNA data sets, including new miRNA target-identification software. Nucleic Acids Res. 42: e139-e139.

Kakrana, A., Li, P., Patel, P., Hammond, R., Anand, D., Mathioni, S., and Meyers, B. (2017). PHASIS?: A computational suite for de novo discovery and characterization of phased, siRNA-generating loci and their miRNA triggers. bioRxiv: 158832.

Kong, J., Martin-Ortigosa, S., Finer, J., Orchard, N., Gunadi, A., Batts, L.A., Thakare, D., Rush,

Kotov, A.A., Adashev, V.E., Godneeva, B.K., Ninova, M., Shatskikh, A.S., Bazylev, S.S., Aravin,

Kumar, S., Stecher, G., Suleski, M., and Hedges, S.B. (2017). TimeTree: A Resource for Timelines, Timetrees, and Divergence Times. Mol. Biol. Evol. 34: 1812-1819.

Langmead, B., Trapnell, C., Pop, M., and Salzberg, S.L. (2009). Ultrafast and memory-efficient

Mathioni, S.M., Kakrana, A., and Meyers, B.C. (2017). Characterization of Plant Small RNAs by Next Generation Sequencing. Curr. Protoc. plant Biol. 2: 39-63. RNA and genomic informatics resources for plants. Plant Physiol. 182: 136-146. 
phasiRNAs in grasses are compositionally distinct from other classes of small RNAs. New Phytol. 220: 851-864.

Patel, P., Mathioni, S., Kakrana, A., Shatkay, H., and Meyers, B.C. (2018b). Reproductive phasiRNAs in grasses are compositionally distinct from other classes of small RNAs. New Phytol. 220: 851-864.

Patel, P., Mathioni, S.M., Hammond, R., Harkess, A.E., Kakrana, A., Arikit, S., Dusia, A., and Meyers, B.C. (2020). Reproductive phasiRNA loci and DICER-LIKE5, but not microRNA loci, diversified in monocotyledonous plants. bioRxiv: 2020.04.25.061721.

Pokhrel, S., Huang, K., Bélanger, S., Caplan, J.L., Kramer, E.M., and Meyers, B.C. (2020). Premeiotic, 21-nucleotide Reproductive PhasiRNAs Emerged in Seed Plants and Diversified in Flowering Plants. bioRxiv: 2020.10.16.341925.

Quinlan, A.R. and Hall, I.M. (2010). BEDTools: A flexible suite of utilities for comparing genomic features. Bioinformatics 26: 841-842.

Schnitzbauer, J., Strauss, M.T., Schlichthaerle, T., Schueder, F., and Jungmann, R. (2017). Super-resolution microscopy with DNA-PAINT. Nat. Protoc. 12: 1198-1228.

Song, X. et al. (2012). Roles of DCL4 and DCL3b in rice phased small RNA biogenesis. Plant J. 69: 462-474.

Teng, C., Zhang, H., Hammond, R., Huang, K., Meyers, B.C., and Walbot, V. (2020). Dicer-like 5 deficiency confers temperature-sensitive male sterility in maize. Nat. Commun. 11: 2912. Version 2-A multiple sequence alignment editor and analysis workbench. Bioinformatics

Xia, R., Chen, C., Pokhrel, S., Ma, W., Huang, K., Patel, P., Wang, F., Xu, J., Liu, Z., Li, J., and Meyers, B.C. (2019). 24-nt reproductive phasiRNAs are broadly present in angiosperms. Nat. Commun. 10: 627.

Xia, R., Ye, S., Liu, Z., Meyers, B.C., and Liu, Z. (2015). Novel and recently evolved microRNA clusters regulate expansive F-BOX gene networks through phased small interfering RNAs in wild diploid strawberry. Plant Physiol. 169: 594-610.

Zhai, J. et al. (2015a). A one precursor one siRNA model for pol IV-dependent siRNA biogenesis. 
$431 \quad$ Cell 163: 445-455.

432 Zhai, J., Zhang, H., Arikit, S., Huang, K., Nan, G.-L., Walbot, V., and Meyers, B.C. (2015b).

433 Spatiotemporally dynamic, cell-type-dependent premeiotic and meiotic phasiRNAs in

434 maize anthers. Proc. Natl. Acad. Sci. U. S. A. 112: 3146-51.

435 


\section{$436 \quad$ Figure Legends}

437 Figure 1. Reproductive 24-nt phasiRNAs triggered by miR2275 are abundant in columbine.

438 A. Alignment of miR2275 family members in columbine. The intensity of blue color denotes the

439 level of conservation, with the darker color indicating a more highly conserved sequence; the 440 consensus sequence is shown with a "sequence logo".

441 B. An example polycistronic cluster of miR2275 members in columbine. The mature and star

442 sequences are marked in yellow and blue respectively. Coordinates of the precursor are

443 indicated below the sequences.

444 C. Abundance of miR2275 members in log10(RP20M) in different tissues of columbine. The

445 yellow gradient arrow at the bottom shows the progression of anther development along

446 different stages.

447 D. Expression of miR2275-triggered 24-nt reproductive phasiRNAs in vegetative and

448 reproductive tissues. The key at right indicates the abundance in units of $\log 2(\mathrm{RP} 20 \mathrm{M})$. Bst

449 indicates bud stage, anth indicates anther. RP20M: reads per twenty million mapped reads

450 (normalized abundance).

452 Figure 2. Non conserved triggers generated 24-nt phasiRNAs in columbine.

453 A. Expression of aco_cand81-triggered 24-nt reproductive phasiRNAs in vegetative and

454 reproductive tissues. The key at right indicates the abundance in units of log2(RP20M). Bst

455 indicates bud stage, anth indicates anther.

456 B. Same as A for miR2118/482 derived 24-PHAS loci.

457 C. Above: Consensus sequence of target sites of aco_cand81, derived from 15 24-PHAS loci.

458 Below: Nucleotide sequence alignment of 15 24-PHAS loci with sequence similarity denoted by 459 the intensity of the blue color indicating that the target site is the only conserved region for all 460 precursors.

461 D. Same as C for miR2118, derived from 22 24-PHAS loci.

462 E. Top: Abundance (in RP10M) of small RNAs in both strands of an example locus padded with 4631000 base pairs, each side. sRNA sizes are denoted by colors, as indicated at top. Bottom: 
464 Phasing score of the same locus; the red dot indicates the highest phased sRNA position. The 465 red arrow denotes cleavage site of aco_cand81.

466 F. Same as E for an example 24-PHAS locus triggered by miR2118 members.

Figure 3. Genome-wide distribution of all triggers and 24-PHAS loci in columbine.

A. Chromosomal positions of miR2275, miR2118/482, and aco_cand81 family members (inner circle, red dots) and their 24-PHAS loci (outer circle, green dots). The outermost circle

471 represents chromosomes from 1 to 7.

472 B. Chromosomal positions and strand-specificity of 24-PHAS loci on chromosome 4 interspersed

473 with genes. The black line represents chromosome 4, and the square boxes above and below

474 the line indicate plus and minus strand-specificity, respectively. The light pink, light purple, and

475 light green boxes represent the 24-PHAS loci triggered by miR2275, aco_cand81, and

476 miR2118/482 family members, respectively. Grey rectangle boxes represent annotated protein-

477 coding genes that intersperse the PHAS clusters, with the total number of interspersed genes

478 indicated in each box.

Figure 4. Trigger miRNAs are enriched in microspore mother cells and tapetal cells of anthers in 481 columbine.

482 A. sRNA-PAINT of all the triggers in pre-meiotic, meiotic and post-meiotic anther stages in 483 columbine. I, II, and III indicate pre-meiotic, meiotic, and post meiotic stages. In each stage, an 484 image of an anther lobe was captured, with the different cell layers following the pattern 485 shown in the schematic diagram of B. White dots in the negative control images were gold 486 fiducials that are pre-coated on the coverslips used for focal panel stabilization during imaging 487 process. Imager buffer with no imager strands was used as negative controls on the same 488 hybridized slides shown for each stage. Scale bar $=10 \mu \mathrm{m}$ for all images.

489 B. Left: schematic diagram of the sRNA-PAINT method applied in a lobe of an anther, showing 490 different cell layers, labeled as follows: E: epidermis, En: endodermis, M: middle layer, T:

491 tapetum, MMC: microspore mother cell. The red dots indicate the sRNAs hybridized to different 492 cell layers. Right: Number of imaging probe binding sites of each trigger in MMC (green), 
493 tapetal cells (blue), and other cell type (grey) in pre-meiotic, meiotic, and post-meiotic stages of

494 anther development. The number of binding sites was calculated as an average from ten

495 measurements in a cell (30 pixels) from different cell layers in each sample using Picasso

496 software.

Figure 5. Reproductive 24-nt phasiRNAs and their triggers in the Rosaceae.

499 A. Expression of 24-nt reproductive phasiRNAs derived by the action of non-conserved triggers,

500 in different tissues and anther development stages in wild strawberry; Athst indicates anther

501 stage. The key at right indicates the abundance in units of log2(RP20M).

502 B. Above: Alignment of sequence logos of miR11308 and target site of miR11308 for six 24-

503 PHAS loci. Below: Nucleotide sequence alignment of six 24-PHAS loci with sequence similarity

504 denoted by the intensity of the blue color showing that the target site is the only conserved

505 region for all the precursors.

506 C. Genome-wide distribution of miR2275, miR11308 and miR2118/482 family members (inner

507 circle, red dots) and their 24-PHAS loci (outer circle, green dots). The outermost circle

508 represents chromosomes from 1 to 7 as numbered, plus chromosome " 8 " which represents an

509 amalgam of the unassembled regions.

510 D. Accumulation of 24-nt reproductive phasiRNAs derived by the action of miR2275, in different

511 tissues and anther development stages in rose. The key at right indicates the abundance in

512 units of log2(RP20M); Bst indicates bud stage, and anth indicates anther.

513 E. Genome-wide distribution of miR2275 family members (inner circle, red dots) and their 24-

514 PHAS loci (outer circle, green dots). The outermost circle represents chromosomes from 1 to 7 ,

515 as numbered.

517 Figure 6. Conservation of reproductive 24-nt phasiRNAs in angiosperms.

518 A. Phylogeny showing the representative orders and species of angiosperms. Only one order

519 and one species from each monophyletic clade of eudicots, monocots, and magnoliids was

520 chosen. Phylogenetic tree was modified from the timetree database. MYA = million years ago.

521 In the table at right, a check mark indicates presence and X indicates absence; NA indicates 'not 
522 analyzed'. Species name from basal angiosperm orders left empty because they lack sequenced

523 genomes for analysis.

524 B. Model for generation of 24-nt reproductive phasiRNAs in angiosperms in terms of trigger

525 diversity and Dicer processing. There are at least three miRNA family members involved in

526 generating 24-nt phasiRNAs in columbine and strawberry. In rose, other eudicots, and

527 monocots, miR2275 is the only trigger. DCL5 is monocot specific, so DCL3 may function in dicing

528 24-nt phasiRNAs in eudicots. 
Table 1. Eudicots confirmed for the presence of 24-nt phasiRNA pathway

\begin{tabular}{|c|c|c|c|c|c|c|c|c|c|c|}
\hline $\begin{array}{l}\text { Species - common } \\
\text { name or shorthand }\end{array}$ & $\begin{array}{l}\text { Scientific } \\
\text { name }\end{array}$ & Family & $\begin{array}{l}\text { Genome } \\
\text { sequenced? }\end{array}$ & $\begin{array}{l}\text { Genome } \\
\text { size }\end{array}$ & $\begin{array}{l}\text { Plant life } \\
\text { cycle }\end{array}$ & $\begin{array}{l}\text { No of } \\
24-n t \\
\text { PHAS } \\
\text { loci }\end{array}$ & $\begin{array}{l}\text { miRNA } \\
\text { triggers of } \\
\text { reproductive } \\
\text { phasiRNAs }\end{array}$ & $\begin{array}{l}\text { No. of } \\
\text { miR2275 } \\
\text { duplexes }\end{array}$ & $\begin{array}{l}\text { Genomic } \\
\text { copies of } D C L 3\end{array}$ & References \\
\hline Columbine & $\begin{array}{l}\text { Aquilegia } \\
\text { coerulea }\end{array}$ & Ranunculaceae & Yes & $\sim 300 \mathrm{Mb}$ & Perennial & 646 & $\begin{array}{l}\text { miR2275, } \\
\text { aco_cand81, } \\
\text { miR2118/482 }\end{array}$ & 15 & 2 & This study \\
\hline Grape & Vitis vinifera & Vitaceae & Yes & $\sim 487 \mathrm{Mb}$ & Perennial & 42 & miR2275 & 10 & 1 & $\begin{array}{l}\text { Xia et al., } \\
2019\end{array}$ \\
\hline Rose & $\begin{array}{l}\text { Rosa } \\
\text { chinensis }\end{array}$ & Rosaceae & Yes & $\sim 503 \mathrm{Mb}$ & Perennial & 112 & miR2275 & 10 & 2 & This study \\
\hline Strawberry & $\begin{array}{l}\text { Fraqaria } \\
\text { vesca }\end{array}$ & Rosaceae & Yes & $\sim 240 \mathrm{Mb}$ & Annual & 335 & $\begin{array}{l}\operatorname{miR} 2275 \\
\operatorname{miR} 11308 \\
\operatorname{miR} 2118 / 482\end{array}$ & 17 & 2 & $\begin{array}{l}\text { Xia et al., } \\
\text { 2019; this } \\
\text { study }\end{array}$ \\
\hline Citrus & $\begin{array}{l}\text { Citrus } \\
\text { sinensis }\end{array}$ & Rutaceae & Yes & $\sim 367 \mathrm{Mb}$ & Perennial & 32 & miR2275 & 10 & 2 & $\begin{array}{l}\text { Xia et al., } \\
2019\end{array}$ \\
\hline Litchi & $\begin{array}{l}\text { Litchi } \\
\text { chinensis }\end{array}$ & Sapindaceae & No & $\begin{array}{l}\sim 550-620 \\
\mathrm{Mb}\end{array}$ & Perennial & 113 & miR2275 & 6 & 2 & $\begin{array}{l}\text { Xia et al., } \\
2019\end{array}$ \\
\hline Cotton & $\begin{array}{l}\text { Gossypium } \\
\text { hirsutum }\end{array}$ & Malvaceae & Yes & $\begin{array}{l}\sim 2,295 \\
\mathrm{Mb}\end{array}$ & Annual & 3 & miR2275 & 1 & 2 & $\begin{array}{l}\text { Xia et al., } \\
2019\end{array}$ \\
\hline Tomato & $\begin{array}{l}\text { Solanum } \\
\text { lyopersicum }\end{array}$ & Solanaceae & Yes & $\sim 950 \mathrm{Mb}$ & Annual & 137 & None & None & 1 & $\begin{array}{l}\text { Xia et al., } \\
2019\end{array}$ \\
\hline Petunia_ax & $\begin{array}{l}\text { Petunia } \\
\text { axillaris }\end{array}$ & Solanaceae & Yes & $\sim 181 \mathrm{Mb}$ & Perennial & 317 & None & None & 1 & $\begin{array}{l}\text { Xia et al., } \\
2019\end{array}$ \\
\hline Petunia_inf & $\begin{array}{l}\text { Petunia } \\
\text { inflata }\end{array}$ & Solanaceae & Yes & $\sim 197 \mathrm{Mb}$ & Perennial & 366 & None & None & 1 & $\begin{array}{l}\text { Xia et al., } \\
2019\end{array}$ \\
\hline
\end{tabular}




\section{Supplemental Figure Legends}

Supplemental Figure 1. The 24-nt phasiRNAs triggered by miR2275 in columbine

A. Above: Sequence logo of target sites of 646 24-PHAS loci triggered by miR2275. Below: Nucleotide sequence alignment of 24-PHAS loci, with sequence similarity indicated by the intensity of the blue color. Even at low resolution, it is apparent that the only target site is conserved.

B. Top: Abundance (RP10M) of small RNAs in both strands of an example locus padded with 1000 base pairs, each side. sRNA sizes are denoted by colors as indicated. Below: Phasing score of same locus and the red dot indicates the highest phased SRNA position. The cleavage site of miR2275 is denoted by the red arrow.

Supplemental Figure 2. Aco-cand81 and miR2118/482 family members and a cluster of 24-PHAS loci triggered by aco_cand81.

A. Alignment of members of the aco_cand81 family of miRNAs in columbine. The degree of conservation is indicated by the intensity of the blue color and the consensus sequence of the alignment is shown as a sequence logo

B. Same as A for miR2118 family members in columbine.

C. A cluster of ten 24-PHAS loci triggered by aco_cand81 in chromosome 4 spread in a $32.2 \mathrm{~kb}$ region. The custom browser shot shows abundances (RP10M) in top and phasing score in below tracks; the light orange region in rectangle grid shows predicted 24-PHAS loci region, and the red arrow indicates the cleavage site.

D. Dot plot of all of the 24-PHAS loci in chromosome 4 in columbine showing similarity within and between PHAS loci in nucleotide level. Green dots indicates $>75 \%$ and light green dots indicates $>50 \%$ similarity.

Supplemental Figure 3. Position-specific biases in the nucleotide composition of 24-nt phasiRNAs and hc-siRNAs in columbine.

A. Profiles of position-specific base usage of each nucleotide ( $A, C, G$ and $U$ ) comparing miR2275-derived 24-nt phasiRNAs and hc-siRNAs in columbine. All of the 24-nt phasiRNAs 
triggered by miR2275 with $>5$ raw reads ( 12000 phasiRNAs) were compared with the 1000 most-abundant hc-siRNAs (matched to transposons and/or repetitive sequences). The open circle in each position indicates frequencies of each of the nucleotides, the small square boxes (highlighted with dotted circle) indicates a statistically significant ( $p=1 e-4)$ position which distinguishes two types of small RNAs.

B. Similar to A, comparing miR2118- and aco_cand81-derived 24-nt phasiRNAs with hc-siRNAs. C. Similar to A, comparing miR2118- and aco_cand81-derived 24-nt phasiRNAs with miR2275derived 24-nt phasiRNAs.

Supplemental Figure 4. 24-nt phasiRNAs triggered by miR2275 in wild strawberry.

A. Accumulation pattern of miR2275-derived 24-nt reproductive phasiRNAs in different tissues and anther development stages of wild strawberry. Athst indicates anther stage. The key at right indicates the abundance in units of log2(RP20M).

B. Alignment of members of miR2275 family in wild strawberry.

C. Abundance of miR2275 members in log10(RP20M) in different tissues of wild strawberry.

Supplemental Figure 5. 24-nt phasiRNAs triggered by miR2275 in rose.

A. A polycistronic precursor of miR2275 from chromosome 5 in rose. The mature and star sequences are indicated by yellow and blue respectively. The names of mature sequence were mentioned near the duplex structure, the grey highlighted names represents non-expressed candidate miR2275 sequences. The chromosomal coordinates of the precursor are indicated below the structure.

B. Alignment of miR2275 mature sequences detected in sRNA sequencing data in rose.

C. Abundance of miR2275 members in log10(RP20M) in different tissues of rose.

\section{Supplemental Figure 6. miR2275 in a magnoliid.}

A. Alignment of unique miR2275 mature sequences detected in genome of Magnolia biondii. B. A polycistronic precursor of miR2275 from contig utg000427I in Magnolia biondii. The mature and star sequences are indicated by yellow and blue respectively. The names of mature 
sequence were mentioned near the duplex structure. The chromosomal coordinates of the precursor are indicated below the structure.

\section{Supplemental Tables}

Supplemental Table S1. miR2275 identified in columbine as triggers of reproductive 24-nt phasiRNAs.

Supplemental Table S2. miR2275, miR2118/482, and aco_cand81 triggered 24-nt reproductive phasiRNAs in columbine.

Supplemental Table S3. miR2275, miR2118/482, and miR11308 triggered 24-nt reproductive phasiRNAs in wild strawberry.

Supplemental Table S4. miR2275 triggered 24-nt reproductive phasiRNAs in rose.

Supplemental Table S5. miR2275 identified in Magnolia biondii and Rosa chinensis as triggers of reproductive 24-nt phasiRNAs.

Supplemental Table S6. Probes designed for in situ hybridization of three triggers of reproductive 24-nt phasiRNAs in Aquilegia. 
A
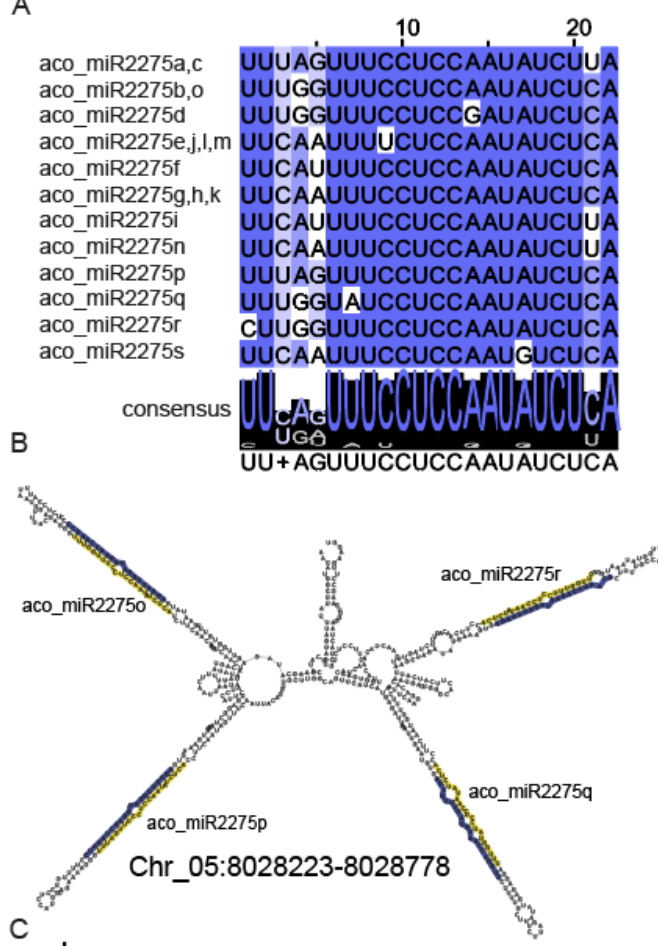

C

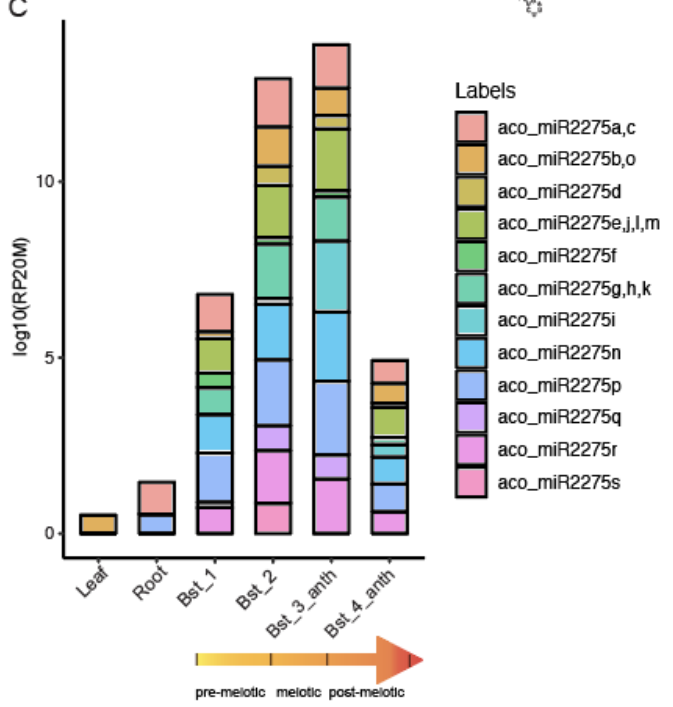

D

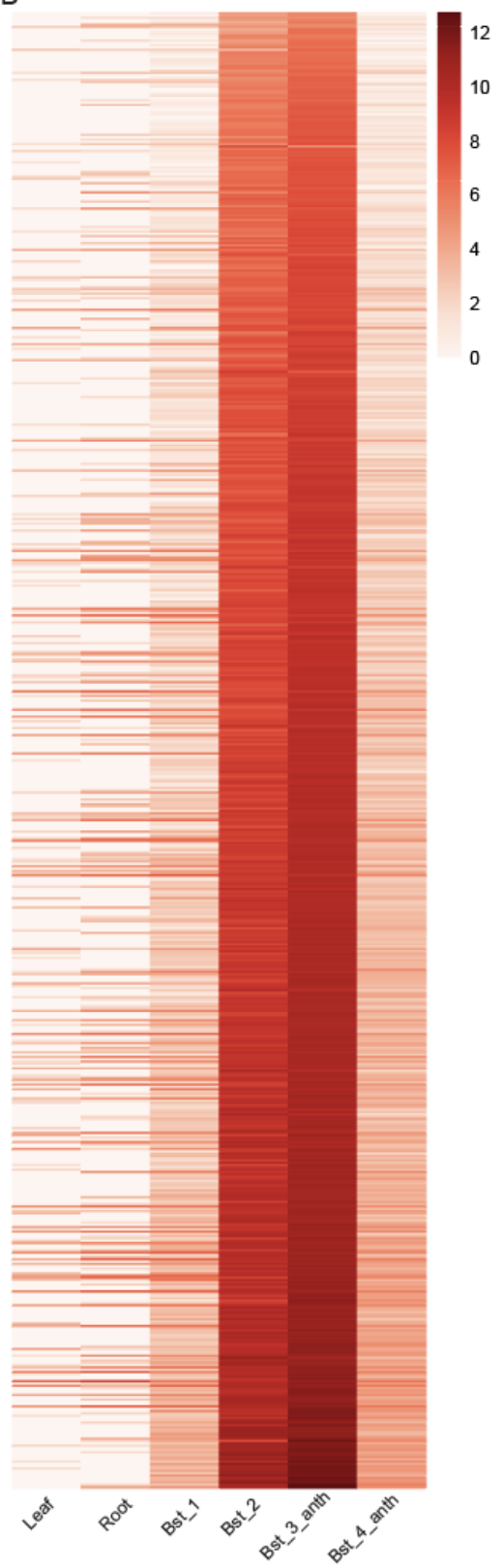

Figure 1. Reproductive 24-nt phasiRNAs triggered by miR2275 are abundant in columbine.

A. Alignment of miR2275 family members in columbine. The intensity of blue color denotes the level of conservation, with the darker color indicating a more highly conserved sequence; the consensus sequence is shown with a "sequence logo". B. An example polycistronic cluster of miR2275 members in columbine. The mature and star sequences are marked in yellow and blue respectively. Coordinates of the precursor are indicated below the sequences. C. Abundance of miR2275 members in log10(RP20M) in different tissues of columbine. The yellow gradient arrow at the bottom shows the progression of anther development along different stages. D. Expression of miR2275-triggered 24-nt reproductive phasiRNAs in vegetative and reproductive tissues. The key at right indicates the abundance in units of log2(RP20M). Bst indicates bud stage, anth indicates anther. RP20M: reads per twenty million mapped reads (normalized abundance). 
A

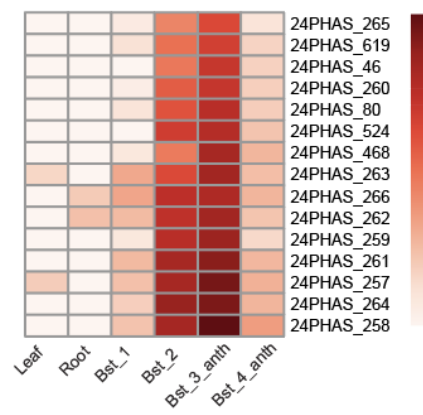

C

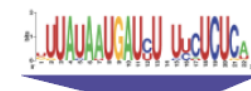

B

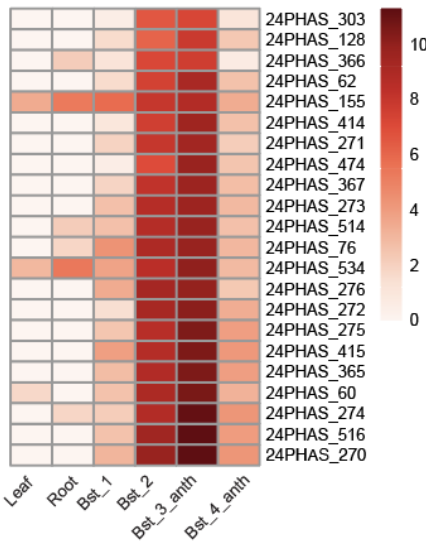

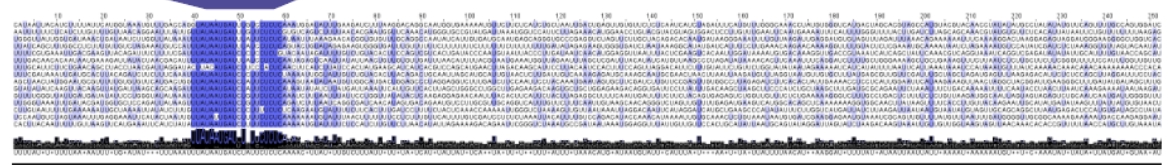

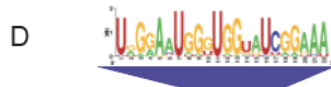

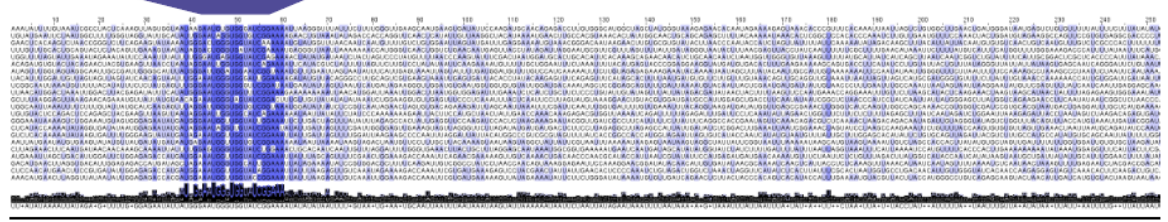

$\mathrm{E}$
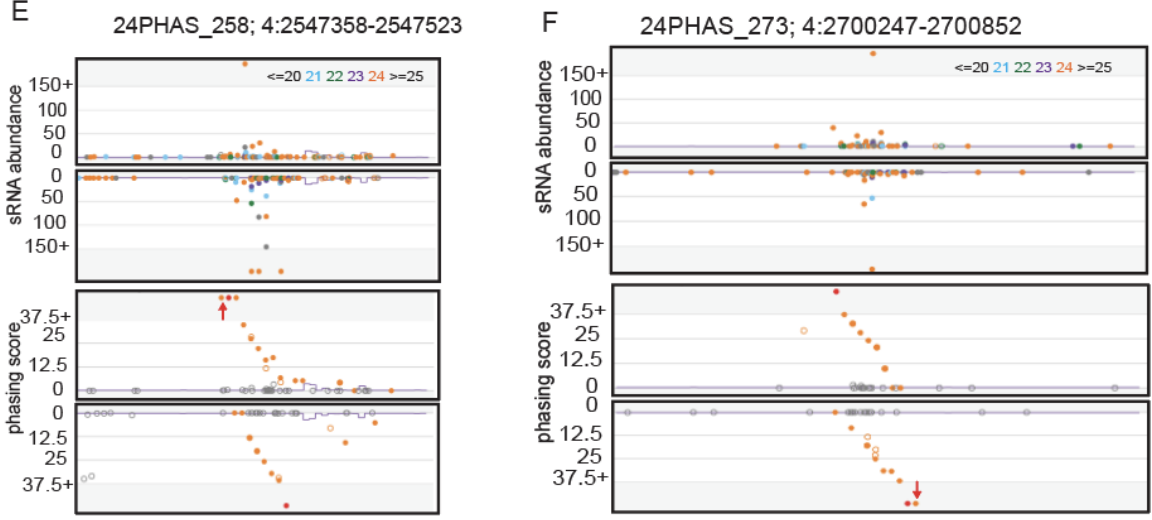

Figure 2. Non-conserved triggers generated 24-nt phasiRNAs in columbine.

A. Expression of aco_cand81-triggered 24-nt reproductive phasiRNAs in vegetative and reproductive tissues. The key at right indicates the abundance in units of log2(RP20M). Bst indicates bud stage, anth indicates anther. B. Same as A for miR2118/482 derived 24-PHAS loci. C. Above: Consensus sequence of target sites of aco-cand81, derived from 15 24-PHAS loci. Below: Nucleotide sequence alignment of $1524-P H A S$ loci with sequence similarity denoted by the intensity of the blue color indicating that the target site is the only conserved region for all precursors. D. Same as C for miR2118, derived from 22 24-PHAS loci. E. Top: Abundance (in RP10M) of small RNAs in both strands of an example locus padded with 1000 base pairs, each side. sRNA sizes are denoted by colors, as indicated at top. Bottom: Phasing score of the same locus; the red dot indicates the highest phased sRNA position. The red arrow denotes cleavage site of aco_cand81. F. Same as E for an example 24-PHAS locus triggered by miR2118 members. 


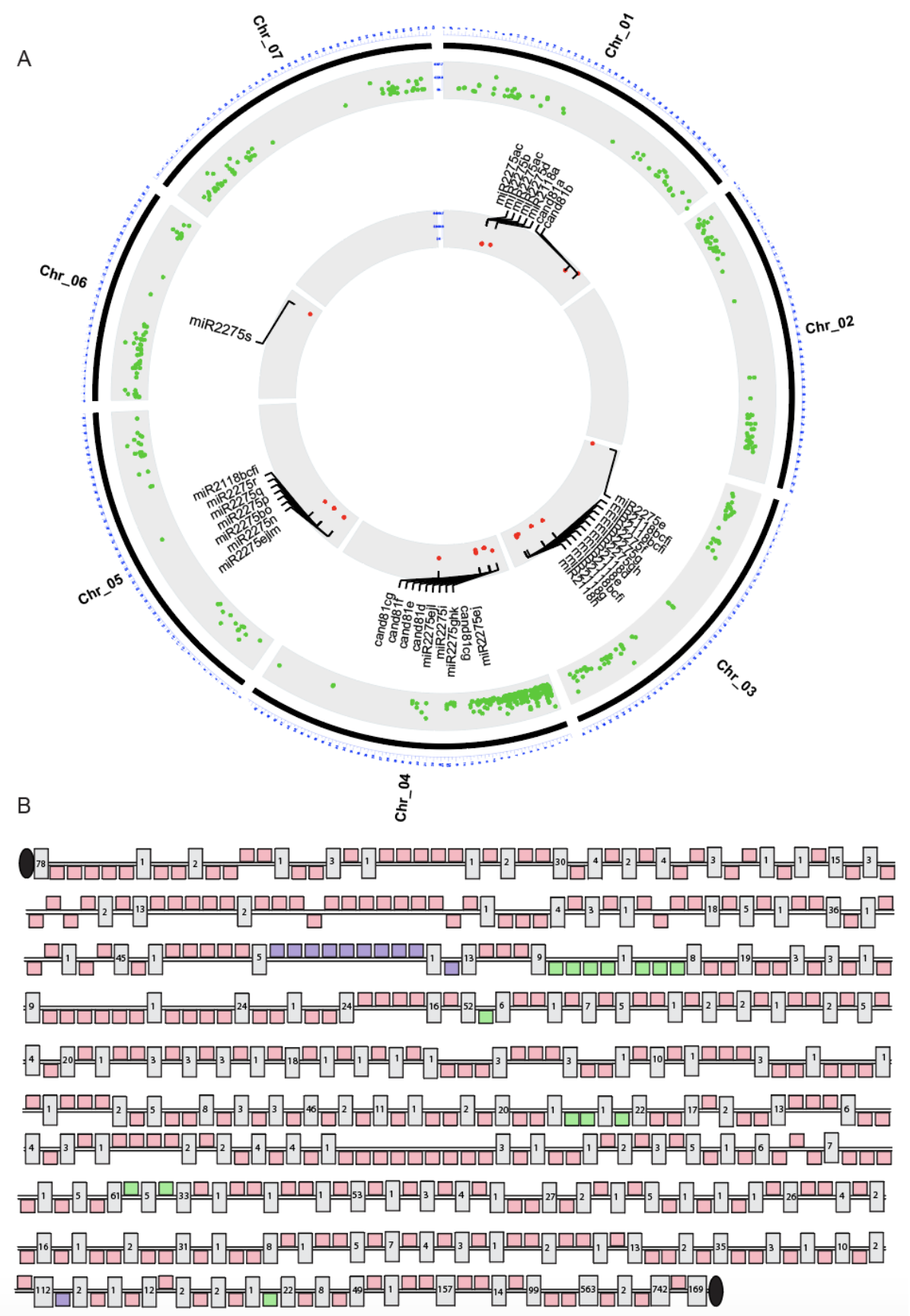

Figure 3. Genome-wide distribution of all triggers and 24-PHAS loci in columbine.

A. Chromosomal positions of miR2275, miR2118/482, and cand81 family members (inner circle, red dots) and their 24-PHAS loci (outer circle, green dots). The outermost circle represents chromosomes from 1 to 7. B. Chromosomal positions and strand-specificity of 24-PHAS loci on chromosome 4 interspersed with genes. The two black lines represent plus and minus strands of chromosome 4, and the square boxes above and below the lines indicate plus and minus strand-specificity, respectively. The light pink, light purple, and light green boxes represent the 24-PHAS loci triggered by miR2275, aco_cand81, and miR2118/482 family members, respectively. Grey rectangle boxes represent annotated protein-coding genes that intersperse the PHAS clusters, with the total number of interspersed genes indicated in each box. The black ovals represent "telomeric end" for chromosome 4. 


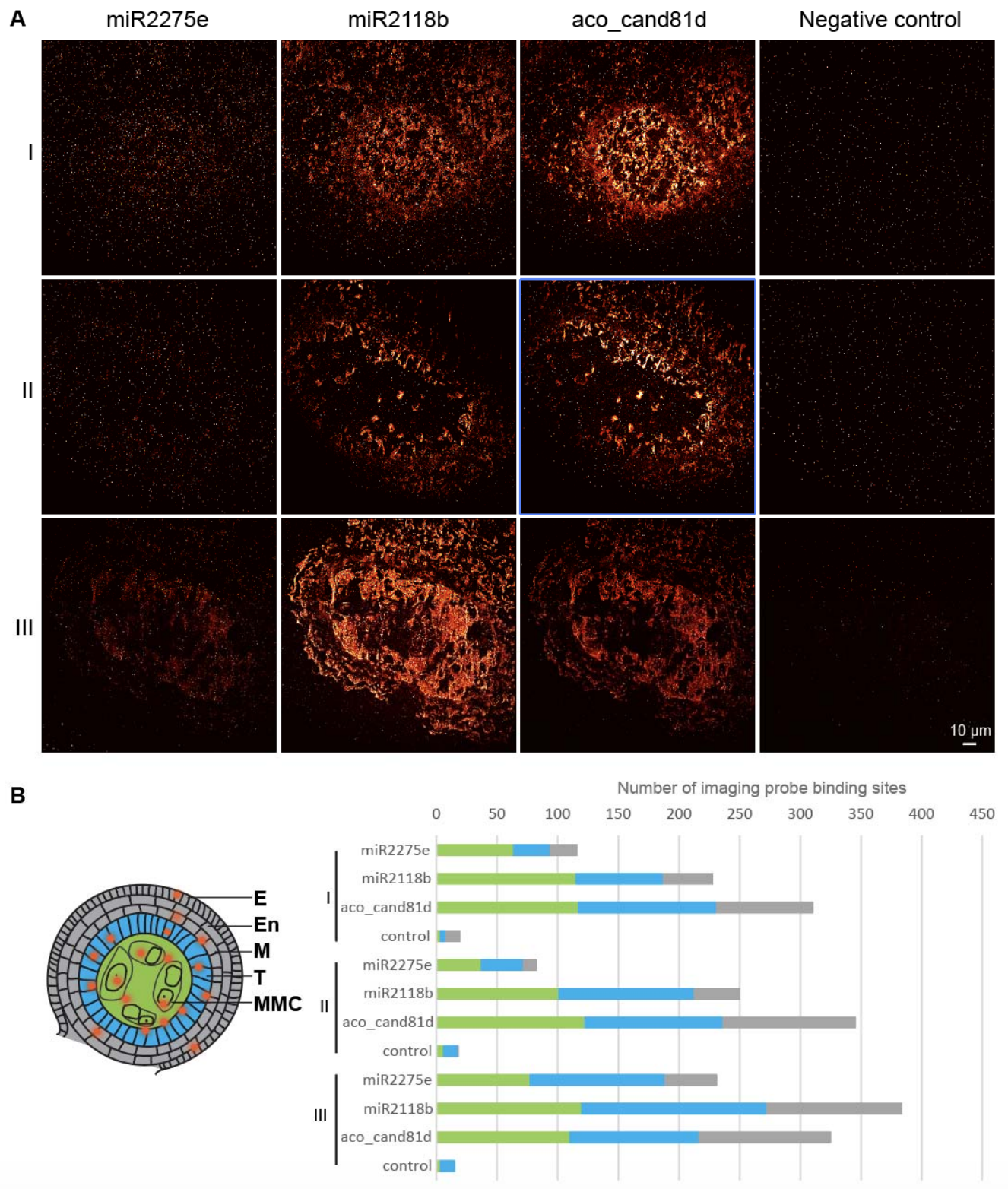

Figure 4. Trigger miRNAs are enriched in microspore mother cells and tapetal cells of anthers in columbine.

A. SRNA-PAINT of all the triggers of reproductive phasiRNAs in pre-meiotic, meiotic and postmeiotic anther stages in columbine. I, II, and III indicate pre-meiotic, meiotic, and post meiotic stages. In each stage, an image of an anther lobe was captured, with the different cell layers following the pattern shown in the schematic diagram of B. B. Left: schematic diagram of the sRNA-PAINT method applied in a lobe of an anther, showing different cell layers, labeled as follows: E: epidermis, En: endodermis, M: middle layer, T: tapetum, MMC: microspore mother cell. The red dots indicate the sRNAs hybridized to different cell layers. Right: Number of imaging probe binding sites of each trigger in MMC (green), tapetal cells (blue), and other cell type (grey) in pre-meiotic, meiotic, and post-meiotic stages of anther development. The number of probe binding sites was calculated as an average from ten measurements in a cell (approx. 30 pixels) from different cell layers in each sample. 

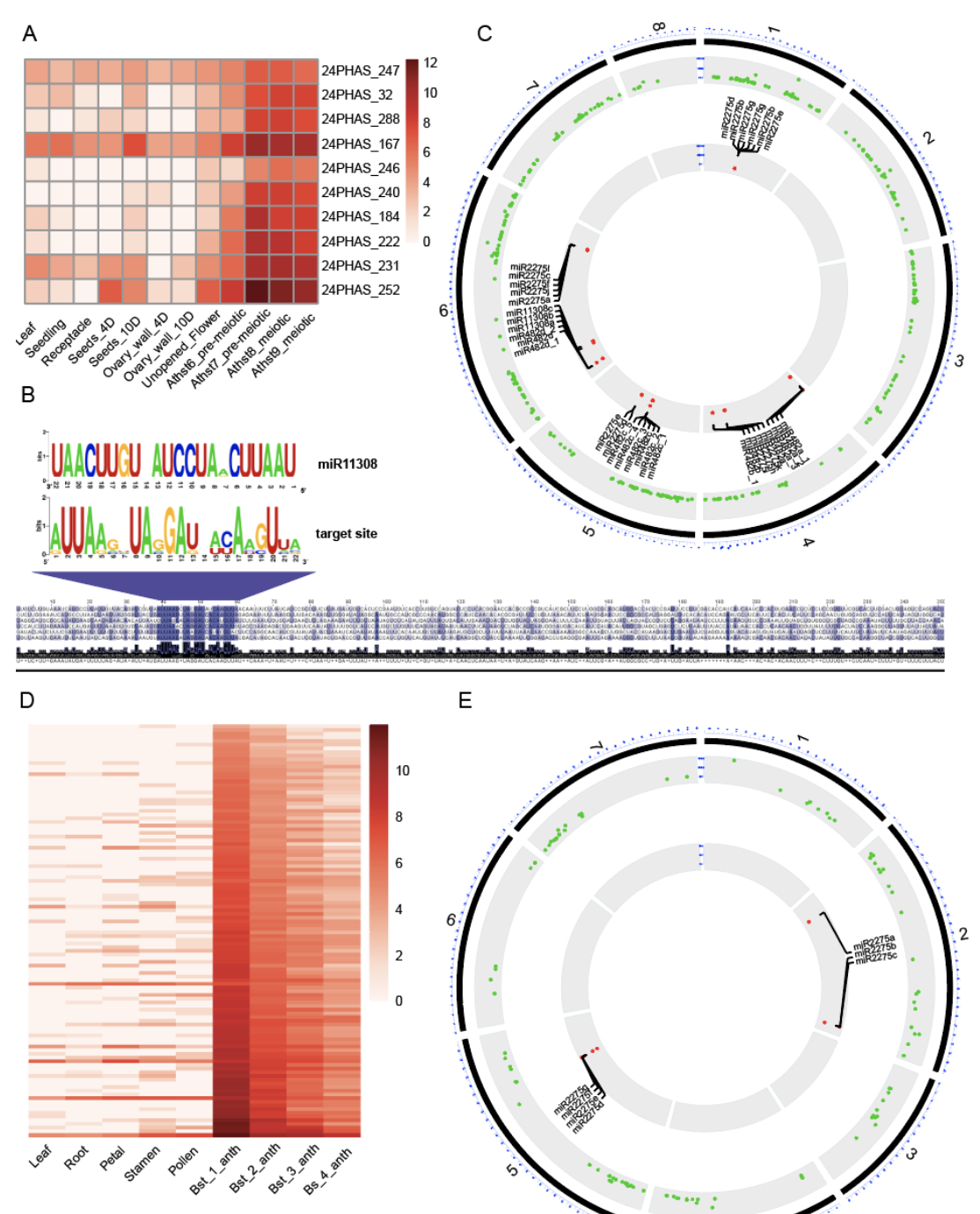

E

Figure 5. Reproductive 24-nt phasiRNAs and their triggers in the Rosaceae.

A. Expression of 24-nt reproductive phasiRNAs derived by the action of non-conserved triggers, in different tissues and anther development stages in wild strawberry; Athst indicates anther stage. The key at right indicates the abundance in units of log2(RP20M). B. Above: Alignment of sequence logos of miR11308 and target site of miR11308 for six 24-PHAS loci. Below:

Nucleotide sequence alignment of six 24-PHAS loci with sequence similarity denoted by the intensity of the blue color showing that the target site is the only conserved region for all the precursors. C. Genome-wide distribution of miR2275, miR11308 and miR2118/482 family members (inner circle, red dots) and their 24-PHAS loci (outer circle, green dots). The outermost circle represents chromosomes from 1 to 7 as numbered, plus chromosome " 8 " which represents an amalgam of the unassembled regions. D. Accumulation of 24-nt reproductive phasiRNAs derived by the action of miR2275, in different tissues and anther development stages in rose. The key at right indicates the abundance in units of $\log 2(\mathrm{RP} 20 \mathrm{M})$; Bst indicates bud stage, and anth indicates anther. E. Genome-wide distribution of miR2275 family members (inner circle, red dots) and their 24-PHAS loci (outer circle, green dots). The outermost circle represents chromosomes from 1 to 7 , as numbered. 
A
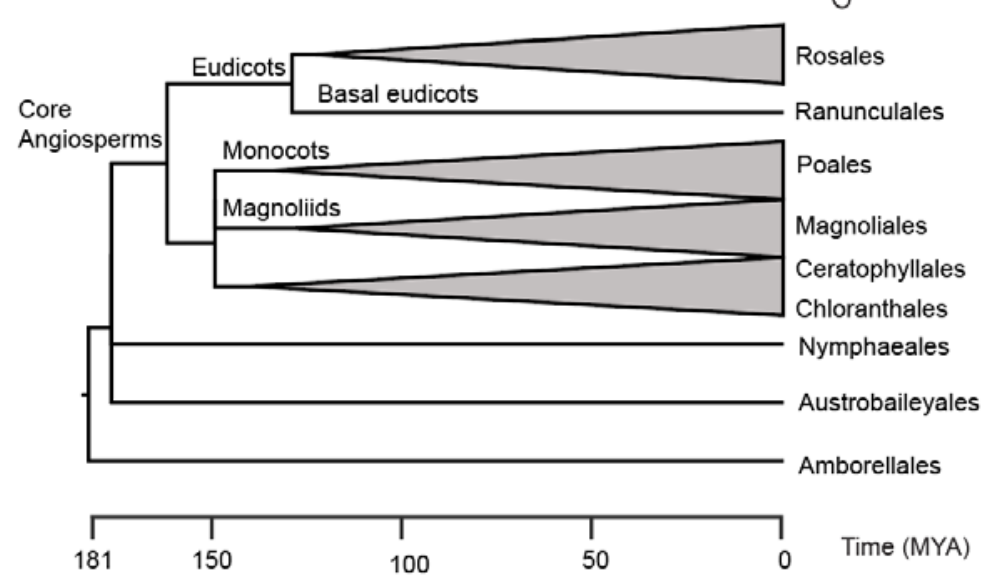

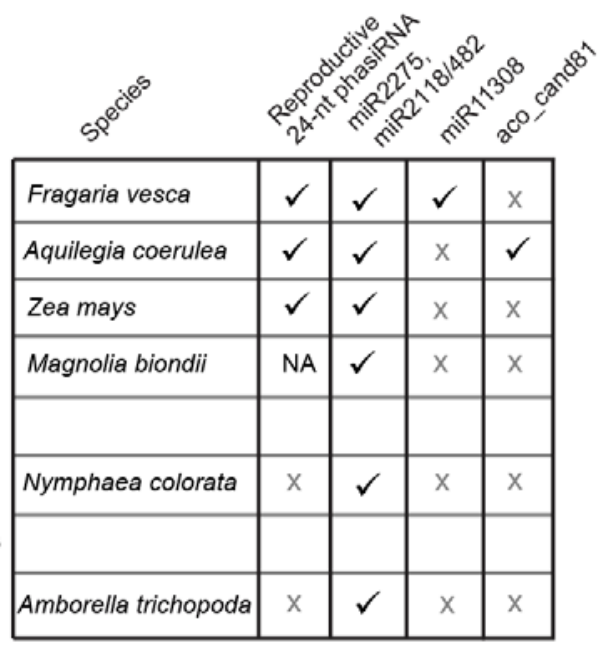

\begin{tabular}{|l|l|l|l|l|}
\hline Amborella trichopoda & $\times$ & $\checkmark$ & $\times$ & $\mathrm{X}$ \\
\hline
\end{tabular}

B

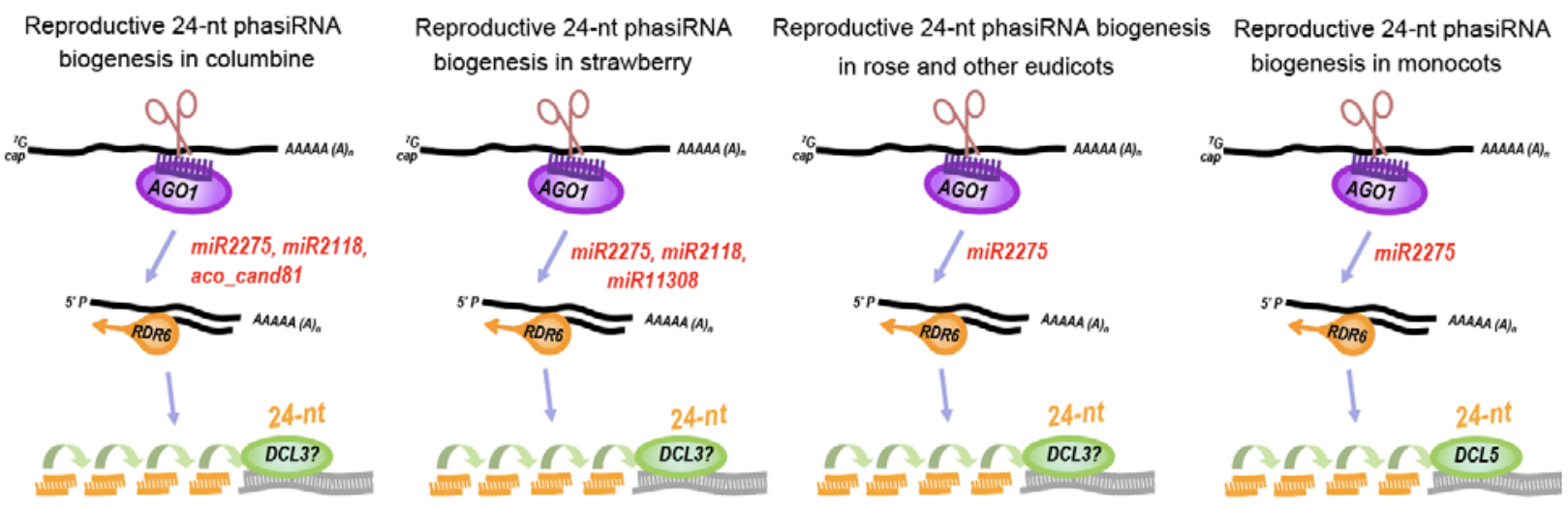

Figure 6. Conservation of reproductive 24-nt phasiRNAs in angiosperms.

A. Phylogeny showing the representative orders and species of angiosperms. Only one order and one species from each monophyletic clade of eudicots, monocots, and magnoliids was chosen. Phylogenetic tree was modified from the timetree database. MYA = million years ago. In the table at right, a check mark indicates presence and $X$ indicates absence; NA indicates 'not analyzed'. Species name from basal angiosperm orders left empty because they lack sequenced genomes for analysis. B. Model for generation of 24-nt reproductive phasiRNAs in angiosperms in terms of trigger diversity and Dicer processing. There are at least three miRNA family members involved in generating 24-nt phasiRNAs in columbine and strawberry. In rose, other eudicots, and monocots, miR2275 is the only trigger. DCL5 is monocot specific, so DCL3 may function in dicing 24-nt phasiRNAs in eudicots. 